\title{
Photovoltaic-Diesel Hybrid System How to Adapt to Real Site Conditions?
}

\author{
Adjat Sudradjata ${ }^{*}$, Eddy Kantosa $^{a}$ \\ ${ }^{a}$ Balai Besar Teknologi Energi (B2TE- BPPT), Gedung 620 PUSPITEK Serpong Tangerang Selatan, Indonesia
}

\begin{abstract}
Integrating the diesel generator with photovoltaic arrays, so called PV-Diesel Hybrid System, have several advantages such as reducing daily operation hour of diesel generator while servicing to the clients increase and the diesel engine is always running on its best efficiencies. However it is required the best system design that complies with the real site conditions which is very different from site to site. The differences are not only the incoming solar radiation but also, the availability of open land for locating the arrays, the conditions of existing diesel engine, and load profile.

The goal of the PV-diesel hybrid system is reducing the diesel operation hours but delivering the electricity for 24 hours to the customers. Enough information of site conditions will guide to proper design of PV-diesel hybrid system. The paper will elaborate the design of PV-diesel hybrid system and how to apply for certain site condition with the existing diesel generator.
\end{abstract}

Keywords: Photovoltaic, Diesel, Hybrid System

\section{Introduction}

The PV-diesel hybrid system is the integration of photovoltaic system with diesel generator to supply the load. The purpose of this technology is providing electricity for 24 hours to the customers but reducing the operation hours of diesel generator in an optimal way. The systems consist of PV arrays, diesel generator, batteries and inverter. The basic operation is controlled by knowing the condition of load and battery.

The application of this technology needs some prerequisites such as, the open land enough for locating the photovoltaic arrays and batteries as an energy storage, and the availability of good condition of diesel generator with automatic start/stop. However; most of the existing diesel are old and there is not enough space for PV arrays which is close to diesel generator. So it has to be a solution that the hybrid technology could adapt to these real situations.

\section{PV-Diesel Hybrid System}

The PV-Diesel hybrid system has to be completed with the control system with the capabilities to run the entire component in a certain conditions. The energy sources from photovoltaic arrays and diesel generator has to be optimum supplying the daily load energy. The block diagram is shown in figure 1 , and basic operation could be seen in figure 2 .

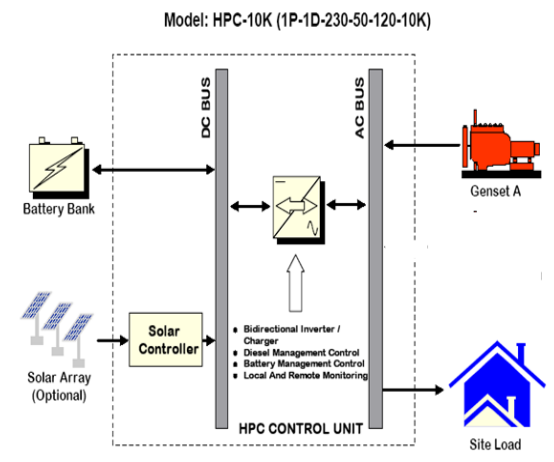

Fig.1 Block diagram of PV-diesel hybrid system

Corresponding Author:
Email: sudradjat_adjat@yahoo.com

ISSN 2413-5453 (C) 2015 The Authors.

Published by KnowledgeE Publishing Services. This is an open access article under the CC BY-NC-ND license (http://creativecommons.org/licenses/by-nc-nd/4.0)

Peer-review under responsibility of EBTKE-CONEX 2013

DOI http://dx.doi.org/10.18502/ken.v1i1.328 
The basic operation of photovoltaic hybrid system could be divided into 3 load conditions, which are low load, medium load and peak load.

Under low load conditions the diesel generator is off and the load energy supplied by the PV energy through the inverter. The diesel generator will operate at optimal loading to feed the load, and it will charge the battery if there is excess energy at medium load. At the peak load condition diesel generator running at optimal loading in parallel with inverter. Inverter is converting $\mathrm{DC}$ power from the battery into $\mathrm{AC}$ power.

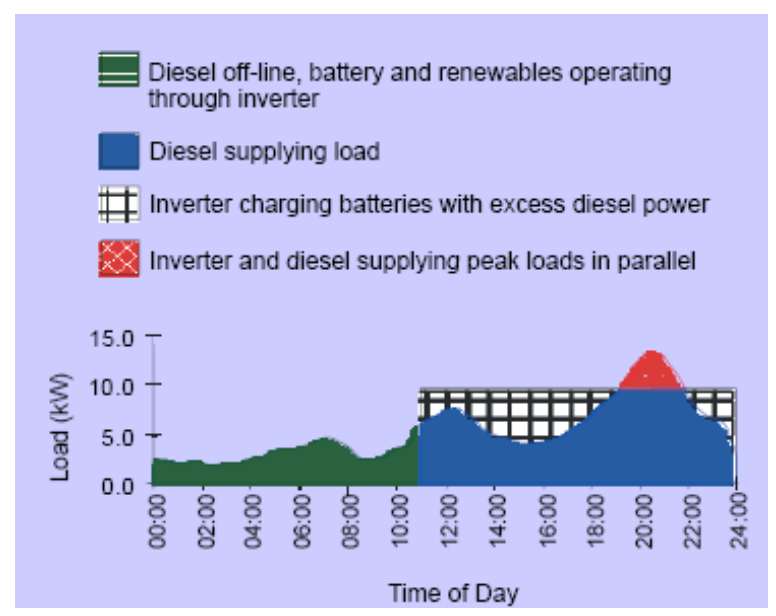

Fig. 2 Typical system operation

\section{Options Mode to adapt to the real site conditions.}

Complete information of site condition is compulsory to design and to implement the PV-diesel hybrid system.

The required information are the availability and location of open space for PV arrays, the daily load profile, how many and what is the capacity, is there any automatic start/stop in existing diesel generator. The design of PV-diesel hybrid system will be based on the above information.

\subsection{Fully Automatic mode.}

Usually the control unit of PV-diesel hybrid system is set with fully automatic, however; most of the diesel generator on site does not have automatic start/stop control. So the operator must start the diesel generator when the control unit trigger and give a sign to the operator to start the diesel generator

The fully automatic mode with the operator has to start the diesel generator by watching the sign from control is too risky, because when the diesel generator has to start in the middle of the night and the operator is not aware, then the system will shut-off.

The characteristic performance of automatic mode can be seen in figure 3 .

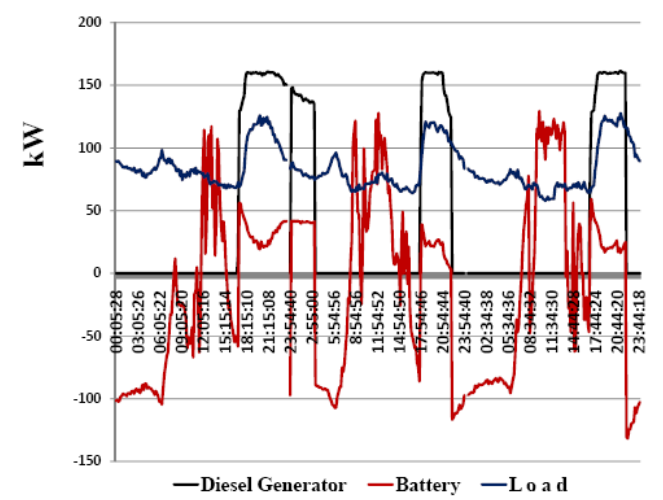

Fig. 3 The characteristic performance of automatic mode

The characteristic performance shows that the load is supplied directly by photovoltaic array during a good solar radiation and the excess energy goes to the battery that will be used for the load at night or at low incoming solar radiation.

When the site load came to a higher than preset level or during low solar radiation, the diesel generator will be started and brought on line in parallel with the inverter to supply the load and/or charge the batteries

\subsection{Run-source (semi automatic) Mode}

The fully automatic mode cannot be performs optimally if the diesel generator do not completed with the automatic start/stop control, because the operator has to switch on and off the diesel generator when the control system ask to do so. Sometime the operator does not switch on or switch off the diesel generator and it will cause the whole system shutdown. To avoid this problem the control unit has to have the run sources mode, which is the running of diesel generator will be depend on the operator. The operator should operate the diesel generator according to a schedule, 
while synchronization between inverter and diesel generator still controlled by the control unit.

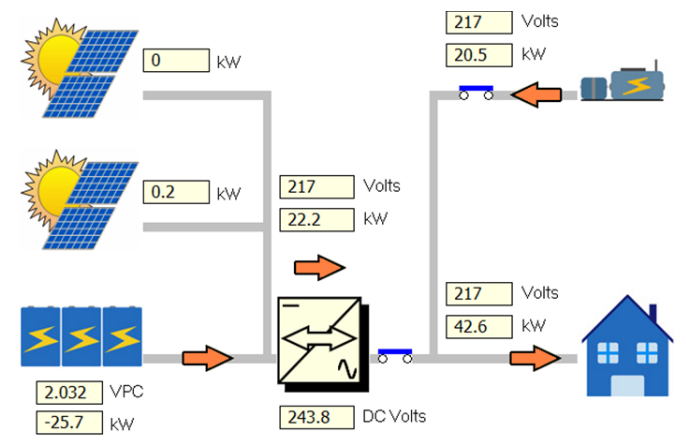

Fig. 4 The block diagram of run source mode

\subsection{Manual Mode.}

The other option is the manual mode. This mode has to be available due to the real facts that so many existing diesel power plants do not have the automatic start/stop control

By using this manual mode it could be set depend on the operation pattern that decide by the system planner.

The usual operation patterns are:

a. All load supplied by diesel generator

b. All load supplied by diesel generator and excess energy goes to charge the battery.

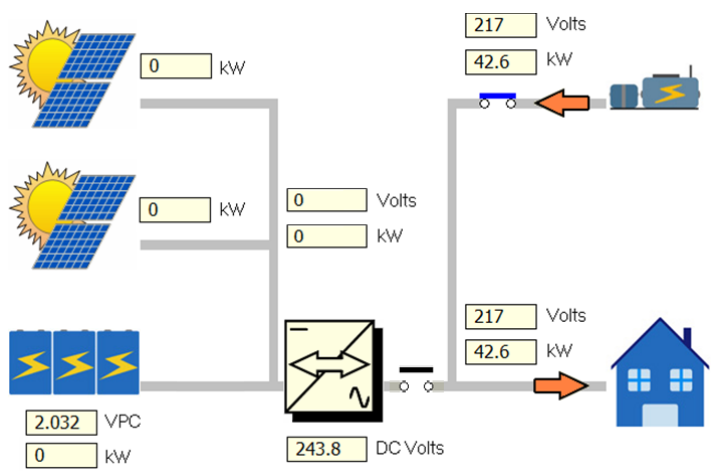

Fig. 5 The block diagram of all load supplied by diesel only

\section{Result and Discussion.}

Most of the real conditions on sites are not supported by hybrid technology. For example that the existing diesel generator is not provided with automatic start/stop control and there is no available open space close to diesel generator where PV arrays should be installed.

Operating system in the fully automatic mode without automatic start/stop control on the existing diesel will not performs optimally, because the system will shut down when the operator does not turn on the diesel generator. It would be better if the system operated in semi automatic/run source mode, which means that diesel generator should be started or stopped by the operator as their schedule while synchronization between inverter and diesel generator still controlled by the control unit.

The other problem on site appear is when there is no open space for the PV arrays close to diesel generator, then the PV arrays has to be located far from the diesel generator. Which means it need cable to connect the PV arrays and control unit which is located at where diesel generator take place. For this condition it could be done by run source mode but the load sharing between inverter and diesel generator will be done by the operator, and there will not be blackout when the diesel generator takes over the load.

The options of manual mode is used when there is a problem in inverter, and system running with diesel only, or during peak load and battery is in low level.

\section{Conclusion}

To adapt to the real site conditions, the technology of PV-diesel hybrid system has to be completed with several optional modes to anticipate different conditions from one site to the other site.

\section{Acknowledgements}

The authors wish to thank Optimal Power Solutions Pty for letting us to use technical and system data information. 


\section{References}

Sudradjat, A. (2009) Five Years Experience on PV-Diesel Hybrid System in Ponelo island. Prosiding Seminar Nasional XIV - FTI-ITS, Surabaya.

Sudradjat, A. \& Kantosa, E. (2006) From Subang to Ponelo Hybrid Photovoltaic-Diesel System in Indonesia, Lessons Learned". $3^{\text {rd }}$ European Conference $P V-H Y B R I D$ and MINI GRID, Aix en Provence, France.

Mitchell, A. et. al. (2011) Optimisation of High Penetration Renewable Energy in Diesel Grids with a view to Zero Diesel Consumption. World Renewable Energy Congress - Indonesia, Bali.

Sihombing, P. and Sudradjat, A. (2011) The Performance of the First Biggest PV-Diesel Hybrid System in Indonesia. World Renewable Energy Congress - Indonesia, Nusa Dua Convention Centre, Bali. 